\author{
Gdańsk 2018, Nr. 39 \\ https://doi.org/10.26881/sgg.2018.39.20 \\ Waldemar Czachur \\ Universität Warschau
}

\title{
Argumentieren in Gedenkreden. Eine linguistische Analyse der deutschen und polnischen Reden zum 25. Jahrestag der Versöhnungsmesse in Kreisau/Krzyżowa
}

Das Ziel des Beitrages ist es, auf die Spezifik des Argumentierens in den Gedenkreden, die sich auf grenzübergreifende, hier die deutsch-polnische Versöhnung beziehen, einzugehen. Es werden in der Arbeit zwei Gedenkreden analysiert, die am 20. November 2014 anlässlich des 25. Jahrestages der deutsch-polnischen Versöhnungsmesse in Kreisau / Krzyżowa von der deutschen sowie der polnischen Regierungschefin gehalten wurden. Im Mittelpunkt steht die Frage, wie die Versöhnungsmesse als Ereignis in beiden Reden sprachlich konzeptualisiert und argumentativ funktionalisiert wurde.

Schlüsselwörter: Gedenkrede, deutsch-polnische Versöhnung, Argumentation, Kreisau/Krzyżowa

Argumentation in commemorative speeches. A linguistic analysis of German and Polish speeches on the 25th anniversary of the reconciliation mass in Kreisau/Krzyżowa. - The aim of the article is to address the specific characteristics of argumentation in commemorative speeches that refer to cross-border reconciliation (here: the German-Polish case). The paper analyses two commemorative speeches given by the German and Polish heads of government on 20th November 2014 on the occasion of the 25th anniversary of the German-Polish reconciliation mass in Kreisau / Krzyżowa. The central question is how the reconciliation mass as an event was conceptualized linguistically and functionalised argumentatively in both speeches.

Keywords: commemorative speech, German-Polish reconciliation, argumentation, Kreisau/Krzyżowa

\section{Vorbemerkungen}

Argumentieren spielt in der politischen Kommunikation eine zentrale Rolle, denn mit Argumenten als Beweismittel versuchen die Politiker/-innen ihre Zielgruppen zu überzeugen, um Macht zu gewinnen oder an der Macht zu bleiben (vgl. Bartoszewicz 2000, Makowski 2013). Politische Reden als ein Bestandteil der politischen Kommunikation lassen sich in dissensorientierte und konsensorientierte Reden klassifizieren (vgl. Klein 2000). Sie zeichnen sich auch durch die Anwendung zahlreicher persuasiver Strategien aus. Die Gedenkreden gelten hingegen als konsensorientierte bzw. epideiktische Textsorte/Redegattung, denn sie thematisieren ein politisch und/oder ethisch relevantes Ereignis aus der Vergangenheit einer Gemeinschaft für die identitätsstiftende Besinnung.

Vor diesem Hintergrund soll in der vorliegenden Arbeit empirisch geprüft werden, wie in Gedenkreden, die am 20. November 2014 anlässlich des 25. Jahrestages der deutsch- 
polnischen Versöhnungsmesse in Kreisau (Krzyżowa), die von der deutschen sowie der polnischen Regierungschefin gehalten wurden, die Versöhnungsmesse als Ereignis sprachlich konzeptualisiert und argumentativ funktionalisiert wurde. Somit versteht sich diese Arbeit als ein Beitrag zur Erforschung der Diskurse zum deutsch-polnischen Dialog (vgl. Bartoszewicz 2000, 2001; Engel 2013, Czachur 2016 a, b und c).

\section{Historischer Hintergrund}

Im Herbst 1989 stattete Bundeskanzler Helmut Kohl als erster westlicher Politiker Polen einen Besuch ab, das damals erst seit Kurzem vom ersten nicht kommunistischen Ministerpräsidenten Tadeusz Mazowiecki regiert wurde. Neben zahlreichen politischen Gesprächen über die deutsche Unterstützung bei den polnischen Reformen wurde am 12. November 1989 ein deutsch-polnischer Gottesdienst auf dem ehemaligen Gut der Familie von Moltke in Kreisau abgehalten. Die Ortsauswahl war vor allem durch die heftige mediale Debatte bedingt, nachdem klar geworden war, dass Kohl eine deutschsprachige Messe mit der deutschen Minderheit am Sankt Annaberg (Góra Świętej Anny) plante. Dies wurde in Polen, aber auch in einigen Kreisen in der Bundesrepublik negativ wahrgenommen, denn am Fuße jenen Berges hatten Polen und Deutsche nach Ende des Ersten Weltkrieges um Schlesien gekämpft, was in Polen als Symbol der Niederlage gilt. Darüber hinaus befürchtete Mazowiecki, dass eine deutschsprachige Messe an einem symbolträchtigen Ort mit der deutschen Minderheit, deren Existenz derzeit immer noch von der kommunistischen Regierung negiert wurde, Konflikte hervorrufen würde, zumal Kohl diesen Aspekt bewusst in den Vordergrund seines Besuches stellte und nicht die Frage der Anerkennung der Oder-Neiße-Grenze oder der Entschädigung für die polnischen Opfer der deutschen Besatzung. Als Alternativort für den deutsch-polnischen Gottesdienst schlug Mazowiecki Kreisau vor und sicherte seine Teilnahme zu. Während der Messe am 12. November 1989, die vom Oppelner Bischof Alfons Nossol gefeiert wurde, tauschten beide Regierungschefs den Friedensgruß aus, der zum Symbol der deutsch-polnischen Versöhnung oder des Neubeginns in den deutsch-polnischen Beziehungen wurde (vgl. Franke/Kretschmann 2016, Czachur 2018b). In den 1990er Jahren wurde der Gebäudekomplex renoviert und eine deutsch-polnische Jugendbegegnungsstätte der Stiftung Kreisau für Europäische Verständigung eingerichtet.

Anlässlich des 25. Jahrestages der Versöhnungsmesse wurde auf dem Gelände der Stiftung Kreisau die Freilichtausstellung „Mut und Versöhnung“ gezeigt, die den deutschpolnischen Annäherungs- und Versöhnungsprozess thematisierte. Sie wurde gemeinsam von der polnischen Ministerpräsidentin Ewa Kopacz und der Bundeskanzlerin Angela Merkel am 20. November 2014 eröffnet. In dem Zusammenhang wurden auch Gedenkreden gehalten, die hier analysiert werden sollen.

\section{Gedenkreden als Textsorte}

Gedenkreden werden im Rahmen von Gedenkveranstaltungen gehalten, um öffentlich an historische Ereignisse zu erinnern, die für politische Gemeinschaften eine sinnstiftende 
Funktion haben (vgl. Klein 2000, Reisigl 2007). Nationale Gedenkfeierlichkeiten zielen darauf ab, „Nationen als vorgestellte Gemeinschaften symbolisch zu re-inszenieren, Nationalstaaten zu rechtfertigen und mit Legitimität auszustatten" (Reisigl 2007: 48). Steht ein grenz- und nationenübergreifendes, binationales Ereignis im Mittelpunkt des Interesses, wie z.B. ein symbolischer Versöhnungsakt, so ändert sich auch die Logik des politischen Handelns.

Als Referenzpunkt für Gedenkreden gilt in dem Zusammenhang ein symbolischer Versöhnungsakt, der mittels sprachlicher und/oder ritueller Handlungen ausgeführt, medial über affektive Bilder distribuiert und Teil des kollektiven Gedächtnisses wurde, wie z.B. der Händedruck von Kohl und Frankreichs Präsident François Mitterrand 1984 in Verdun oder der Friedensgruß zwischen Mazowiecki und Kohl 1989 in Kreisau. Mit dem bilateralen Begehen eines Gedenktages signalisieren die politischen Entscheidungsträger einvernehmlich den politischen und gesellschaftlichen Willen, diesem eine sinnstiftende und identitätstragende Funktion in politischen und erinnerungskulturellen Diskursen beizumessen. So werden Versöhnungsakte im kollektiven Gedächtnis beider Gemeinschaften im Rahmen der Jahrestage aktiviert und durch verbale und nonverbale Praktiken jeweils funktionalisiert. Im Idealfall ist diese Funktion dieser Versöhnung in beiden Erinnerungsgesellschaften ähnlich oder vergleichbar, wie z.B. die deutsch-französische Versöhnung als Abkehr von Erbfeindschaft und als Gründungsmythos der Europäischen Union (vgl. Czachur 2018a).

In Anlehnung an Reisigl kann davon ausgegangen werden, dass die Aufgabe einer Gedenkrede, auch in den internationalen Beziehungen darin besteht,

„eine konsensuelle, das heißt intersubjektive, überparteiliche Beurteilung von geschichtlichen und politischen Daten, Ereignissen und Persönlichkeiten vorzugeben, gemeinschafts- bzw. nationsbezogene Normen laudativ oder vituperativ zu sanktionieren und eine einheitliche kognitive, emotionale und volitive Ausrichtung herbeizureden, welche den Wegweiser für das spätere politische Tun der Angesprochenen abgeben soll“ (Reisigl 2007: 51).

Für diese Zwecke ist das Argumentieren von zentraler Bedeutung, weil damit der Geltungsanspruch einer oder mehrerer Thesen kommunikativ untermauert wird. Damit hat das Argumentieren ,immer auch eine appellative und entscheidungsorientierende Funktion“ (Lüger 2017: 242). Um die Akzeptanzbedingungen für die Argumente zu verbessern, werden zusätzliche argumentationsspezifische Mittel eingesetzt, wie das argumentative Entkräften von Gegenthesen, das Anführen zusätzlicher Erklärungen, die gezielte Verwendung von Einstellungskundgaben sowie nicht-argumentationsspezifischen Strategien, wie die der Polarisierung, Emotionalisierung oder der Einsatz von Metaphorik, expressiver Lexik, phraseologischer Ausdrücke, durch nähesprachliche Syntax, Topoi oder rhetorische Figuren (vgl. Lüger 2013, 2017: 242). Beim Argumentieren werden also Sachverhalte miteinander in Beziehung gebracht, durch Reliefgebung/Hervorhebung perspektiviert und durch Bewertungen eingeschätzt (vgl. Engel 2013). Grundsätzlich wird davon ausgegangen, dass die Analyse der argumentativen Struktur in bestimmten Texten dazu genutzt werden kann, die kollektiven Wissensbestände von Sprach- und Kulturgemeinschaften zu erschließen (vgl. Wengeler 2003, Miller 2014) 


\section{Analyse}

In diesem Teil der Arbeit soll die argumentative Struktur der oben genannten Reden analysiert werden, um die sprachliche Konzeptualisierung der Versöhnungsmesse sowie ihre argumentative Funktionszuweisung erfassen zu können. Zunächst wird hier der inhaltliche Aufbau beider Reden betrachtet sowie ihre Hauptthesen und die unterstützenden Argumente erschlossen, um anschließend die Funktion der deutsch-polnischen Versöhnung, die in den untersuchten Reden konstruiert wird, aufzuzeigen.

Dabei werden die jeweiligen thematischen Sequenzen der Rede betrachtet, die im Text miteinander verknüpft sind und als Ganzheit ein argumentatives Potenzial darstellen.

Tab.1: Inhaltlicher Aufbau der Rede von Merkel und Kopacz

\begin{tabular}{|l|l|}
\hline \multicolumn{1}{|c|}{$\begin{array}{c}\text { Die Rede von Bundeskanzlerin Angela Merkel } \\
\text { (1066 Wörter) }\end{array}$} & \multicolumn{1}{|c|}{$\begin{array}{c}\text { Die Rede von Polens Ministerpräsidentin } \\
\text { Ewa Kopacz (633 Wörter) }\end{array}$} \\
\hline $\begin{array}{l}\text { Vor dem Hintergrund der Feierlichkeiten anläss- } \\
\text { lich des Berliner Mauerfalls werden der Beitrag der } \\
\text { Polinnen und Polen beim Zerfall des Kommunismus } \\
\text { sowie die Ängste vor einem vereinigtem Deutschland } \\
\text { thematisiert. }\end{array}$ & $\begin{array}{l}\text { Die Versöhnungsmesse, ihr politischer Kon- } \\
\text { text, die Bedeutung des Friedensgrußes sowie } \\
\text { die Akteure des Ereignisses werden charakterisiert. }\end{array}$ \\
\hline $\begin{array}{l}\text { In Bezug auf den Zweiten Weltkrieg werden das pol- } \\
\text { nische Leid, die deutsche Verantwortung sowie Ver- } \\
\text { treibung als Konsequenz thematisiert. }\end{array}$ & $\begin{array}{l}\text { Der deutsch-polnischen Interessengemeinschaft aus } \\
\text { dem Jahr 1989 wird explizit die Fähigkeit aus der } \\
\text { Geschichte zu lernen, zugeschrieben. Und es wer- } \\
\text { den die Erfolge deutsch-polnischer Institutionen } \\
\text { sowie Netzwerke auf vielen Ebenen thematisiert. }\end{array}$ \\
\hline $\begin{array}{l}\text { Die Versöhnungsmesse wird im Kontext des } \\
\text { deutsch-polnischen Versöhnungsprozesses charak- } \\
\text { terisiert, ihre Bedeutung, ihre Akteure spezifiziert } \\
\text { und Kreisau als symbolischer Ort hervorgehoben. }\end{array}$ & $\begin{array}{l}\text { Im Hinblick auf Erfolge wird auf die Notwen- } \\
\text { digkeit der faktenbasierten Wahrheit im Rahmen } \\
\text { der Geschichtsvermittlung in beiden Ländern } \\
\text { hingewiesen. }\end{array}$ \\
\hline $\begin{array}{l}\text { Er werden politische und wirtschaftliche Erfolge } \\
\text { in den deutsch-polnischen Beziehungen erwähnt } \\
\text { und die aktuellen gemeinsamen Interessen und } \\
\text { Werte, auch im Hinblick auf die sicherheitspoliti- } \\
\text { schen Besorgnisse der polnischen Bürger/-innen, } \\
\text { thematisiert. }\end{array}$ & $\begin{array}{l}\text { Der Mut polnischer und deutscher Individuen } \\
\text { und ihr Wille zur Wirklichkeitsveränderung wird } \\
\text { hervorgehoben. }\end{array}$ \\
\hline $\begin{array}{l}\text { Vor dem Hintergrund der Lehren aus der Geschichte } \\
\text { wird für die Verteidigung des partnerschaftlichen } \\
\text { Lebens in einem friedlichen Europa plädiert und } \\
\text { hervorgehoben werden die deutsch-polnischen } \\
\text { Erfahrungen von vor 25 Jahren als Beispiel für } \\
\text { Wirklichkeitsveränderungen. }\end{array}$ & \\
\hline
\end{tabular}

Aus dieser Zusammenstellung wird ersichtlich, dass in den beiden Reden einige Sequenzen identisch sind, wie die Charakterisierung der Versöhnungsmesse, ihres politischen Kontextes, der Bedeutung des Friedensgrußes sowie der Akteure des Ereignisses, aber auch die Charakterisierung der Erfolge im deutsch-polnischen Dialog. Für die deutsche Rede lässt sich 
folgende Struktur identifizieren: Benennung/Feststellung des geschichtlichen Kontextes mit dem Verweis auf die Rolle Polens und Deutschlands [der polnische Kampf gegen den Kommunismus und auf deutscher Seite die Übernahme von Verantwortung für den Zweiten Weltkrieg], Bewertung der Versöhnungsmesse, der Erfolge in den deutsch-polnischen Beziehungen sowie der aktuellen politischen Herausforderungen [der Umgang mit Russland] sowie der Appell für die Verteidigung eines friedlichen Europas. Für die polnische Rede lässt sich folgende Struktur ermitteln: Bewertung der Versöhnungsmesse sowie der Erfolge in den deutsch-polnischen Beziehungen und der Appell für eine fundierte Geschichtsvermittlung und ein entschlossenes Handeln gegen Geschichtsfälschung.

Die Erfassung der argumentativen Struktur in politischen Reden ist deswegen nicht immer leicht und eindeutig, weil sie mehrfachadressiert wird und bei heterogenen Zielgruppen unterschiedliche Erwartungen erfüllt werden müssen. Darüber hinaus ergibt sich die Eignung einer Äußerung als Argument „nicht zuletzt daraus, ob oder inwieweit der Empfänger einen logischen bzw. regelhaften Bezug zur problematisierten These herstellen kann" (Lüger 2017: 247). Dies wird aber auch dadurch erschwert, dass die Sachverhalte in vielen, auch den untersuchten Reden implizit und nicht mittels entsprechender Konnektoren zur Herstellung der Kausalität, Adversativität, Konditionalität, Konsekutivität oder Finalität relationiert werden (vgl. Kątny 2013). Ein hermeneutisches Verfahren ist hier gefragt.

Zunächst soll die argumentative Struktur in der Rede von Merkel anhand eines Redeausschnittes analysiert werden. Hierzu ein Beleg:

[1] „Meine Damen und Herren, die Vergangenheit lehrt uns, dass unser Weg in Europa ein Weg der Partnerschaft und des friedlichen Ausgleichs der Interessen sein muss. [2] Wir Europäer sind heute, wie wir es auch am 50. Jahrestag der Unterzeichnung der Römischen Verträge 2007 formuliert haben, zu unserem Glück vereint. [3] Wir müssen dieses Glück immer wieder verteidigen. [4] Ich bin sehr froh, dass wir dies gemeinsam tun - auch weil wir die wunderbare Erfahrung teilen, die wir vor 25 Jahren gemacht haben: Veränderung zum Guten ist möglich. [5] Dies sollte uns auch in unserem weiteren Zusammenwirken anspornen - zum Wohle unserer beiden Nationen, zum Wohle Europas und seiner Menschen."

Es handelt sich hier um den abschließenden Abschnitt der Rede. Aus der Äußerung [3 und 4] kann folgende These abgeleitet werden: Wir [Europäer, implizit: darunter Deutsche und Polen] können Veränderung zum Guten herbeiführen. Diese These wird durch Argumente unterschiedlicher Art untermauert, wie:

- Die Polen haben 1989 den Anderen, auch den DDR-Bürgerinnen/Bürgern, Mut gegeben für die Freiheit zu kämpfen (ethisches Argument);

- Die Deutschen ziehen eine Lehre aus der Geschichte des Zweiten Weltkrieges und übernehmen hierfür Verantwortung (ethisches Argument);

- Erinnerung an die Verbrechen und Versöhnung sind wichtig, die Versöhnungsmesse war ein Meilenstein auf diesem Weg (historisch-empirisches Argument);

- Deutschland und Polen haben in den vergangenen 25 Jahren mehr erreicht als man sich hätte träumen lassen (pragmatisches Argument);

- Deutsche und Polen übernehmen gemeinsame Verantwortung in und für Europa (deontisches Argument); 
- Europa steht für Partnerschaft und einen friedlichen Interessenausgleich (pragmatisches Argument).

In der Rede von Kopacz lassen sich zwei Ausschnitte finden, in denen die Hauptthese deutlich wird:

[6] „Zdarza się, że szlachetne gesty, dzięki którym wydobywamy z siebie to, co w nas najlepsze prowadzą do bardzo ważnych zmian. [7] Przekazanie sobie znaku pokoju przez Tadeusza Mazowieckiego i Helmuta Kohla stało się mocnym znakiem woli pojednania i woli tworzenia nowych lepszych relacji między Polakami i Niemcami. [...]”.

[8] „Cieszę się, że możemy dzisiaj z szacunkiem odnieść się do aktów odwagi sprzed dwudziestu pięciu lat, które swą wielką mocą zdołały przemienić rzeczywistość. [9] Myślę tu zwłaszcza o tych, którzy zobaczyli w Niemcach czy Polakach partnera, z którym można i należy podjąć dialog”.

[6] „Es kommt vor, dass edle Gesten, dank derer wir das Beste aus uns herausholen, zu sehr wichtigen Veränderungen führen. [7] Der Friedensgruß zwischen Tadeusz Mazowiecki und Helmut Kohl wurde zu einem starken Zeichen des Willens, sich zu versöhnen und neue und bessere Beziehungen zwischen Polen und Deutschen zu schaffen. [...]“

[8] „Ich bin froh, dass wir heute respektvoll auf die mutigen Taten von vor 25 Jahren verweisen können, die es mit ihrer großen Kraft geschafft haben, die Realität zu verändern. [9] Ich denke insbesondere an diejenigen, die in Deutschland oder in Polen einen Partner gesehen haben, mit dem wir einen Dialog führen können und sollten."

Aus den Äußerung [6] und [8] lässt sich die folgende These ableiten: Edle und mutige Gesten bzw. Taten führen zu wichtigen Veränderungen. Diese These wird durch Argumente unterschiedlicher Art untermauert, wie:

- Der Friedensgruß zwischen Mazowiecki und Kohl war ethisch motiviert und markierte den Beginn eines neuen Kapitels in den deutsch-polnischen Beziehungen (deontisches Argument);

- Polen lernte aus der Vergangenheit und sah in der Wiedervereinigung Deutschlands eine Chance auf die Westanbindung (ethisches Argument);

- Die deutsch-polnische Versöhnung ist eine Summe der Leistungen vieler Menschen (pragmatisches Argument);

- Polen und Deutsche haben gemeinsam viel erreicht (pragmatisches Argument);

- Europa ist das wichtigste Ziel und Argument für partnerschaftliche bilaterale Beziehungen (deontisches Argument);

- Polen und Deutsche sind Nachbarn, Partner und Freunde (deontisches Argument);

- Polen und Deutsche sind in der Verantwortung, ein fundiertes historisches Wissen bereitzustellen, insbesondere der jungen und sich entschlossen gegen die Geschichtsfälschung zu engagieren (deontisches Argument). 
Die beiden oben genannten Thesen werden in den Reden durch ethische, pragmatische, deontische Argumente unterschiedlicher Art gestützt. Diese Argumente werden in beiden Reden vor allem in Form von Behauptungen und Bewertungen realisiert.

In der deutschen Rede stehen Handelnde im Vordergrund, also Europäer/-innen, darunter Polinnen, Polen und Deutsche. Sie können Veränderungen mit europaweiter Relevanz initiieren. Im Mittelpunkt der Rede der polnischen Ministerpräsidentin stehen die edlen Gesten und Taten. Die Handelnden werden hier implizit als Ausführer/-innen der Gesten und Taten konzipiert [9]. Darüber hinaus werden in den Aussagen, die die zentralen Thesen mitgestalten, Ausdrücke, wie: Veränderung, das Gute, Gesten, Taten verwendet, die inhaltlich unmarkiert sind. Ihre Bedeutung weist daher einen universellen und keinen spezifischen politischen Charakter auf.

Interessant ist auch, wie das Ereignis, hier die Versöhnungsmesse sprachlich konzeptualisiert wird, d.h. welche Prädikate als Bewertungsmittel dem zentralen Ereignis zugeschrieben werden. Anhand eines Ausschnitts der Rede von Kopacz soll dieser Prozess veranschaulicht werden:

[9] „Tu w Krzyżowej 12 listopada 1989 roku spotkali się szefowie rządów polskiego i niemieckiego. [10] Spotkali się podczas mszy świętej odprawianej przez obecnego tu dzisiaj arcybiskupa Alfonsa Nosola. [11] Tadeusz Mazowiecki i Helmut Kohl przekazali sobie wtedy znak pokoju. [12] Wydarzenie to mocno wpisało się w historię tamtych dni i zyskało miano mszy pojednania. [13] Trzy dni wcześniej wszyscy to pamiętamy runął mur berliński, a w Europie środkowej rozpoczą się demontaż komunizmu. [14] Wiemy już, że tamto spotkanie stało się początkiem nowego rozdziału w relacjach między naszymi sąsiedzkimi krajami. [...] [15] Przekazanie sobie znaku pokoju przez Tadeusza Mazowieckiego i Helmuta Kohla stało się mocnym znakiem woli pojednania i woli tworzenia nowych lepszych relacji między Polakami i Niemcami. [...] [16] W tamtym momencie gest obu polityków miał przede wszystkim wymiar etyczny. [...] [17] I za tymi wydarzeniami o charakterze etycznym poszly dobre zmiany społeczne i polityczne w stosunkach polsko-niemieckich".

[9] „Hier in Kreisau traffen sich am 12. November 1989 die Regierungschefs Polens und Deutschlands. [10] Sie trafen sich während der Messe, die vom Erzbischof Alphons Nossol gefeiert wurde, der heute hier anwesend ist. [11] Tadeusz Mazowiecki und Helmut Kohl tauschten gegenseitig einen Friedensgruß aus. [12] Dieses Ereignis war fest in die Geschichte jener Tage eingeschrieben und wurde Versöhnungsmesse genannt. [13] Drei Tage zuvor, wir erinnern uns alle daran, ist die Berliner Mauer gefallen, und in Mitteleuropa begann der Abbau des Kommunismus. [14] Wir wissen bereits, dass das Treffen den Beginn eines neuen Kapitels in den Beziehungen zwischen unseren Nachbarländern markierte. [...] [15] Der Friedensgruß zwischen Tadeusz Mazowiecki und Helmut Kohl wurde zu einem starken Zeichen des Willens, sich zu versöhnen und neue und bessere Beziehungen zwischen Polen und Deutschen zu schaffen. [...] [16] Damals hatte die Geste der beiden Politiker in erster Linie eine ethische Dimension. [...] [17] Und diesen ethischen Ereignissen folgten gute gesellschaftliche und politische Veränderungen in den deutsch-polnischen Beziehungen."

Zunächst erfolgt durch die Feststellungen die Kontextualisierung des Ereignisses. Es werden das Ereignis selbst, sein Datum, Ort und Akteure benannt [9, 10, 11, 12]. Das Ereignis selbst wird als Versöhnungsmesse bezeichnet und in einen breiteren historischen Kontext gestellt, den der Öffnung der Berliner Mauer [13]. Erst nach diesen Feststellungen, die eine repräsentative Funktion haben, erfolgt die Bewertung der Versöhnungsmesse, die wiederum ein deklaratives und expressives Potenzial aufweist [14]. Die Versöhnungsmesse wird als 
Beginn eines neuen Kapitels in den nachbarschaftlichen Beziehungen beider Länder und der Friedensgruß als ein starkes Zeichen für den Willen zur Versöhnung und Verbesserung der bilateralen Beziehungen konstituiert.

Anhand eines Ausschnitts der Rede Merkels soll nun dasselbe Phänomen analysiert werden:

[18] „Drei Tage nach dem Mauerfall in Berlin fand hier in Kreisau die Versöhnungsmesse statt, an der Bundeskanzler Helmut Kohl und der polnische Ministerpräsident Tadeusz Mazowiecki gemeinsam teilnahmen. [19] Diese Versöhnungsmesse war ein Meilenstein auf einem Weg, der zuvor mühsam beschritten wurde. [...] [20] Erst mit dem Ende des Kalten Krieges und dem Fall der Berliner Mauer öffnete sich die Tür zu einer in der Tat umfassenden Versöhnung. [21] Helmut Kohl und Tadeusz Mazowiecki trafen sich hier in Kreisau, an einem Ort des deutschen Widerstands und des freien Worts. [22] Mitten im Zweiten Weltkrieg wurde hier über die Nachkriegsvision eines freien Europas gleichberechtigter Völker diskutiert. [23] Kreisau - dieser Name steht für Verständigung, Versöhnung und Partnerschaft. [24] Die Versöhnungsmesse drei Tage nach dem Mauerfall in Berlin hat diesen Geist in jener historischen Stunde unmittelbar spürbar gemacht."

Auch in dieser Rede wird das Ereignis zunächst kontextualisiert und im breiten politischen Kontext des Jahres 1989 positioniert [18]. Die Konzeptualisierung der Versöhnungsmesse als Meilenstein auf dem Weg der Versöhnung wird - ähnlich wie in der Rede von Kopacz - durch die Umbruchsdynamik des Jahres 1989 spezifiziert [19]. Darüber hinaus wird der deutsche Widerstand als Teil der Ortshistorie hervorgebracht [22] und im Sinne der deutschen Geschichtspolitik als eine Referenz stilisiert. Das Erbe des Widerstandes und der Versöhnungsmesse werden in den Begriffen Verständigung, Versöhnung und Partnerschaft synthetisiert. Damit werden die beiden Erfahrungen universalisiert und die Werte des deutschen Widerstandes werden implizit als Fundament der deutsch-polnischen Versöhnung gedeutet. Dieser Aspekt wurde in der Rede der polnischen Ministerpräsidentin nicht thematisiert. Dies ist auch auf eine bestimmte politische Distanz der Polen dem deutschen Widerstand gegenüber zurückzuführen (vgl. Miodek 2013).

\section{Zusammenfassung}

Aus der Analyse der beiden Gedenkreden von Kopacz und Merkel, die 2014 anlässlich des 25. Jahrestages der deutsch-polnischen Versöhnungsmesse in Kreisau gehalten wurden, geht hervor, dass Gedenkreden eine laudative, konsolatorische sowie promissorische Funktion haben und im deutsch-polnischen Vergleich argumentationsstrategisch und sprachlich-konzeptionell - wie die analysierten Abschnitte zeigen - nach demselben Muster konstruiert und ausgeführt werden.

Im Mittelpunkt beider Reden steht der Wille zu Veränderungen als notwendige Voraussetzung für politische und gesellschaftliche Umbrüche und Umgestaltungen. Das Jahr 1989 brachte Deutschland die Einheit und Polen die Freiheit vom Kommunismus und veränderte somit ganz Europa. Dies manifestiert sich auch in dem in beiden Aussagen vorhandenen Begriff Veränderung. Damit wird deutlich, dass die deutsch-polnische Versöhnung hier universalistisch gedeutet wird und ihr binationales Potenzial gar nicht politisch für beide Gesellschaften funktionalisiert wird. Zwar werden in beiden Reden Begriffe wie Versöbnung, 
Verständigung, Partnerschaft, Partner, Freunde, Nachbar verwendet, jedoch spielen sie in der Argumentation eine zweitrangige Funktion.

Interessant sind auch die Wir-Konstruktionen in den beiden Reden. In der polnischen Rede wird konsequent von „wir“ im Sinne von „wir Deutsche und Polen/Polinnen“ gesprochen. In der deutschen Rede lassen sich drei Typen von „Wir-Konstruktionen“ identifizieren: „wir" als Deutsche im Kontext der Wende 1989 sowie des Zweiten Weltkriegs, „wir“ als Deutsche und Polen/Polinnen im Kontext der Versöhnungsmesse und der bilateralen Erfolge sowie „wir" als Europäer/-innen im Appell für die Verteidigung des Friedens in Europa. Auffallend ist, dass diese Wir-Konstruktionen im Sinne eines exklusiven Wir mehrheitlich durch das Pronomen Wir und nur sporadisch durch Ethnonyme Deutsche und Polen konstruiert wird.

In beiden Reden wird die Versöhnungsmesse als ein wichtiges Ereignis im deutsch-polnischen Annäherungsprozess gedeutet. Ihre Funktion im bilateralen Kontext wird sprachlich als „Meilenstein“ und als „Beginn eines neuen Kapitels“ spezifiziert und auch argumentativ als Ereignis von binationaler Relevanz konzeptualisiert. Vergleicht man diese beiden Reden mit denen, die z.B. 1990 während der Unterzeichnung des deutsch-polnischen Nachbarschaftsvertrags von dem polnischen Ministerpräsident Jan Krzysztof Bielecki und dem Bundeskanzler Helmut Kohl gehalten wurden, so fällt hier auf, dass dem deutsch-polnischen Dialog bzw. der deutsch-polnischen Versöhnung nach 25 Jahren keine europaweite Funktion zugeschrieben wird. Anders als 1990 kommt hier der Topos der besonderen Rolle stabiler Beziehungen zwischen Deutschland und Polen für den Frieden in Europa (vgl. Bartoszewicz 2000) gar nicht zur Sprache. Für die weitere Auseinandersetzung mit diesem Thema sowie die fundierte Bewertung dieser Sachverhalte wäre es sinnvoll, Gedenkreden in anderen bilateralen Kontexten, vor allem im deutsch-französischen Zusammenhang zu untersuchen.

\section{Literatur}

Bartoszewicz, Iwona (2000): Formen der Persuasion im deutsch-polnischen politischen Dialog. Untersuchungen zu politischen Reden zwischen 1989 und 1995. Wrocław: Wydawnictwo Uniwersytetu Wrocławskiego.

Bartoszewicz, Iwona (2001): Rhetorik der deutsch-polnischen Versöhnung 1989-1995. In: Studia Linguistica 20, 11-29.

Czachur, Waldemar (2016a): Erinnerungsrhetorische Musterbildungen im deutsch-polnischen Erinnerungsdialog. Eine linguistische Analyse anhand der Reden von deutschen Politikern in den Jahren 1989-2016. In: Dargiewicz, Anna (Hg.): Anfang. Sprachwissenschaftliche Implikationen. Würzburg: Königshausen \& Neumann, 81-95.

Czachur, Waldemar (2016b): Symbolisches Gleichgewicht und internationales Beziehungsmanagement. Eine linguistische Analyse des Briefes der polnischen Bischöfe an die deutschen Bischöfe aus dem Jahre 1965. In: Bąk, Paweł / Rolek, Bogusława (Hg.): Vom Wort zum Gebrauch. Wortbedeutung und ibre Eingebundenheit in Diskurse. Frankfurt/M.: Peter Lang, 261-277.

Czachur, Waldemar (2016c): Mediale Profilierung und kollektives Gedächtnis. Der 25. Jahrestag der Versöhnungsmesse von Krzyżowa / Kreisau im Spiegel deutscher und polnischer Fernsehnachrichten. In: Kaczmarek, Dorota (Hg.): Politik - Medien - Sprache. Deutsche und polnische Realien aus linguistischer Sicht. Łódź: Wydawnictwo Uniwersytetu Łódzkiego, 81-102. 
Czachur, Waldemar (2018a): Versöhnung als multimodaler Erinnerungstext. Eine kultur- und medienlinguistische Analyse am Beispiel der deutsch-französischen und der deutsch-polnischen Beziehungen. In: Czachur, Waldemar / Lüger, Heinz-Helmut (Hg.): Kollektives Gedächtnis und europäische Nachbarschaftsbilder. Beiträge aus kulturlinguistischer Perspektive. Landau: Verlag Empirische Pädagogik, 23-62.

Czachur, Waldemar (2018b): Die Versöhnungsmesse in Kreisau - Resultat eines faulen Kompromisses in der deutsch-polnischen Geschichtspolitik? In: Ziemer, Klaus / Andrychowicz-Skrzeba, Joanna (Hg.): Jenseits der Jubiläen. Geschichtspolitik im deutsch-polnischen Alltag. Warszawa: Friedrich-Ebert-Stiftung, 219-230.

Engel, Ulrich (2013) (Hg.): Argumentieren. Sprechen im deutsch-polnischen Kontrast. Wrocław / Dresden: Atut / Neisse Verlag.

Engel, Ulrich / Tomiczek, Eugeniusz (2010): Wie wir reden. Sprechen im deutsch-polnischen Kontrast. Wrocław / Dresden: Atut / Neisse Verlag.

Franke, Annemarie / Kretschmann, Dominik (2016): Der Friedensgruß von Kreisau 1989. Eine Geste als Versprechen. In: Defrance, Corine / Pfeil, Ulrich (Hg.): Verständigung und Versöhnung nach dem "Zivilisationsbruch"? Deutschland in Europa nach 1945. Bonn: Bundeszentrale für politische Bildung, 137-155.

Kaczmarek, Dorota (2016): Politischer Antidialog - Kommunikation zwischen Abweichung und Norm. In: Convivium. Germanistisches Jahrbuch Polen 2016, 163-190.

Kątny, Andrzej (2008): Bewertendes Einschätzen aus kommunikativer und kontrastiver Sicht. In: Czachur, Waldemar / Czyżewska, Marta (Hg.): Vom Wort zum Text. Studien zur deutschen Sprache und Kultur. Festschrift für Professor Józef Wiktorowicz zum 65. Geburtstag. Warszawa: Instytut Germanistyki UW, 185-192.

Kątny, Andrzej (2013): Äußerungen relationieren. In: Engel, Ulrich (Hg.): Argumentieren. Sprechen im deutsch-polnischen Kontrast. Wrocław / Dresden: Atut / Neisse Verlag, 15-29.

Klein, Josef (2000): Textsorten im Bereich politscher Institutionen. In: Brinker, Klaus / Antos, Gerd / Heinemann, Wolfgang / Sager, Sven F. (Hg.): Text-und Gesprächslinguistik. 1. Band. Textlinguistik. Berlin / New York: de Gruyter, 731-755.

Lüger, Heinz-Helmut (2013): Akzeptanzwerbung in der politischen Rede. Zum Sprachgebrauch der NPD. In: Zeitschrift des Verbandes Polnischer Germanisten 2, 117-127.

Lüger, Heinz-Helmut (2017): Einzeltextorientierte Argumentationsanalyse. In: Niehr, Thomas / Kilian, Jörg / Wengeler, Martin (Hg.): Handbuch Sprache und Politik, Bd.1. Bremen: Hempen, 241-260.

Makowski, Jacek (2013): Die Abgeordnetenrede im Europäischen Parlament. Korpusgestützte textsortenorientierte Analyse deutschsprachiger Wortmeldungen in den Plenardebatten des Europäischen Parlaments. Łódź: Primum Verbum.

Miller, Dorota (2014): Emotionalität und Wertung im Diskurs. Eine kontrastive Analyse deutscher und polnischer Pressetexte zum EU-Beitritt Polens. Frankfurt/M.: Peter Lang.

Miodek, Marcin (2013): Der „Kreisauer Kreis” im polnischen kollektiven Bewusstsein über den deutschen Widerstand gegen den Nationalsozialismus. In: Czachur, Waldemar / Franke, Annemarie (Hg.): Kreisau/Krzyżowa - ein Ort des deutsch-polnischen Dialogs. Herausforderungen für ein europäisches Narrativ. Krzyżowa: Fundacja „Krzyżowa” dla Porozumienia Europejskiego, 96-109.

Reisigl, Martin (2007): Nationale Rhetorik in Fest-und Gedenkreden. Eine diskursanalytische Studie zum „österreichischen Millennium“ in den Jahren 1946 und 1996. Tübingen: Stauffenburg.

Wengeler, Martin (2003): Topos und Diskurs. Begründung einer argumentationsanalytischen Methode und ihre Anwendung auf den Migrationsdiskurs (1960-1985). Tübingen: Niemeyer. 